\title{
Genetic Algorithm-Based Optimization of 3rd Order Multiple Feedback Low Pass Filter
}

\author{
A. A. Wan and S. C. Neoh
}

\begin{abstract}
Modern IC design industry nowadays demands a lot of engineers to produce circuit design with certain specification of outputs. In order to achieve these specifications, the input parameters need to be optimized. Conventional circuit parameter tuning involves trial and error adjustment which required large amount of time to achieve desired specifications. Furthermore, to build up filter circuits that meeting specification requires experience and knowledge about filter characteristic itself. Intense mathematical knowledge in terms of filter performance calculation might be necessary. This paper proposed Genetic Algorithm (GA) based optimization method for optimizing the design variables of the $3^{\text {rd }}$ order multiple feedback low pass filter. The $3^{\text {rd }}$ order multiple feedback low pass filter is synthesized using LTspiceIV whereas the GA model is developed using MATLAB. There are eight design variables to be optimized for achieving the required output gain, pass-band ripple, and targeted cut-off frequency.
\end{abstract}

Index Terms-Genetic algorithm, optimization, multiple feedback, low pass filter.

\section{INTRODUCTION}

Nowadays, analog IC design is highly demanded in the IC industries. The complexity of modern IC design as well as market's demands to produce optimal parameters and high performance design rapidly cause circuit design to be computationally expensive [1]. In $3^{\text {rd }}$ Order Multiple Feedback Low Pass Filter design, the main specifications that need to achieve are gain, cut-off frequency and pass-band ripple. Conventionally, parameters of the circuit design are fine-tuned using trial and error adjustment to achieve the desired specifications which required large amount of time [2]. Besides, the non-linear factor of the circuit which is sensitive to the parameter changes leads to complex trade-off among the required specifications [1]. As a result, appropriate approach of parameter optimization is important to the circuit design industries.

Filter design requires knowledge about filter characteristic and it involves intense mathematical calculation in terms of filter performance [1]. In this research, Genetic Algorithm (GA) that predicated on the concept of nature evolution is applied to solve complex filter parameter optimization problem. The algorithmic development of GA is simple and it is able to efficiently reduce the probing of solution space [3].

Manuscript received May 9, 2013; revised July 13, 2013.

The authors are with School of Microelectronic Engineering, Universiti Malaysia Perlis, Perlis, Malaysia (e-mail: eyradagreat90@yahoo.com, scneoh@unimap.edu).

\section{BACKGROUND}

\section{A. Genetic Algorithm}

Genetic Algorithm (GA) is an intelligent optimization techniques with powerful global searching ability based on the principle of natural evolution where organism produce a number of offspring similar to themselves but can have variation due to mutation (random changes) and crossover (offspring have combination of features inherited from each parent). GA is initially developed by John Holland and also known as adaptive or reproductive method.

Holland's original GA is known as the simple GA (SGA). It provides efficient technique for optimization and machine-learning applications widely applicable to business, science and engineering. GA is one of the many examples of evolutionary Algorithm (EA) that used to optimize the circuit problems [3]. The common techniques used in GA are inheritance, mutation, selection and crossover.

The basic mechanism of GA is designed to simulate the process of evolution in natural system specifically for those that follow the principle of survival of the fittest, first laid by Charles Darwin. In the process of genetic reproduction, some offspring survive and are produced to the next generation and some don't. The probability of survival is based on the individual fitness in which the fitter it is, the higher the survival chance it is.

GA is best used when the objective function is discontinuous, highly non-linear, stochastic or undefined derivatives. GA can provide solutions for highly complex search spaces and it perform well to all types of problems because they do not make any assumption about underlying fitness landscape (the shape of the fitness function, or objective function)

Candidate solutions in GA are represented as chromosomes (strings of genes) in a population. The number of genes is equal to the number of total candidate elements that are available and each gene can be encoded in the form of binary or real value [4]. GA focuses on the mechanism of recombination and mutation as the main evolution engines. The recombination in GA is defined as the exchange of the information between two parents to produce two offspring. It is usually represented by crossover operator which takes some information of both parents and exchanges among themselves. On the other hand, the mutation operator is used by randomly changing some individual's information. The chance of chromosomes to be selected for recombination and mutation mechanism is based on its fitness value in which the better the fitness value, the higher the chance to be selected [3], [5]. 
GA has a lot of benefits and advantages such as the concept is easy to understand, it supports multi-objective optimization, available for parallel run, applicable for hybrid application, and etc. Although GA may consume more time to reach optimal solution when large search space is involved, it is capable of quickly finding promising regions of the search space. In addition, there are lots of application that used GA such as optimization, robotics, machine learning, signal processing, automatic programming, economics, ecology, population genetics and many more.

\section{B. Third Order Multiple Feedback Low Pass Filter}

Multiple feedback topology is an electronic topology for low- pass, high-pass, and band-pass filters. Low pass filters are designed to stop subsonic signals and can be useful to hi-fi applications and sound reinforcement [6]. A low-pass filter is a filter that passes only low-frequency signal according to the cut-off frequency. Fig. 1 shows the schematic diagram of a $3^{\text {rd }}$ order multiple feedback low pass filter. This design is obtained from LTspiceIV and FilterPro. The $3^{\text {rd }}$ order multiple feedback low pass filter can be built by cascading two building blocks made of first order low pass filter and second order low pass filter. For first order low pass filter stage needs two capacitors while for second order low pass filter stage needs three capacitors to get a second-order response. This circuit consists of capacitors $C 5$ and $C 6$ that are set to $47 \mathrm{pF}$ and $C 4$ that is set to $10 \mathrm{nF}$. According to [7], circuit performance is highly affected by the design components. In this circuit, the main design variables of concern are capacitors, $C 1$ and $C 3$, and resistors, $R 1, R 2, R 3$, $R 4, R 5$ and $R 6$. Altogether, there are eight design variables to be optimized. These variables are tuned within the specification range given to achieve the required results or objectives. The design variable range specification for the $3^{\text {rd }}$ order multiple feedback low pass filter are given in Table I. These design variables are to be tuned to achieve the required specifications of gain, pass-band ripple and cut-off frequency shown in Table II. The optimization of this circuit design using GA approach is to maximize the gain (relative magnitude of the output voltage compared to the input voltage), minimize the pass-band ripple (small fluctuations in pass-band) and achieve the cut-off frequency around the range of $1500 \mathrm{~Hz}$ to $1600 \mathrm{~Hz}$.

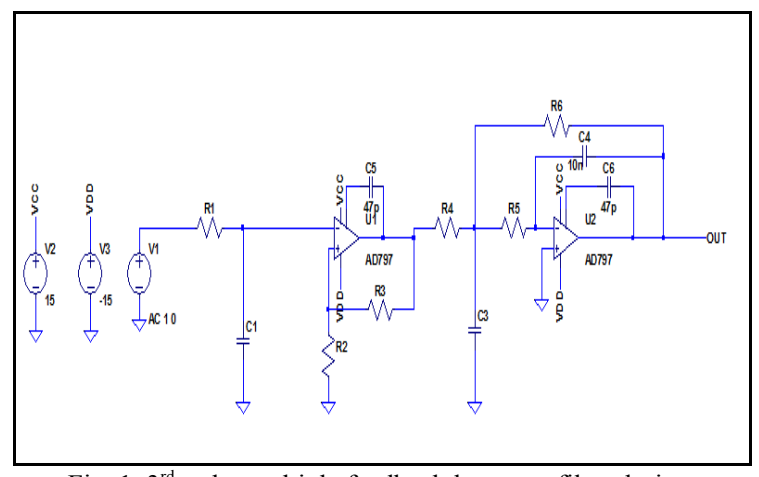

Fig. 1. $3^{\text {rd }}$ order multiple feedback low pass filter design.

\section{OPTIMIZATION}

The GA mechanism is developed in Matlab whereas the filter circuit is simulated in LTspiceIV which is able to perform various electronic circuit simulations and the representation of the simulation's output is clear. The $3^{\text {rd }}$ order multiple feedback low pass filter is tuned according to the parameter setups and design constraints given. Fig. 2 shows the flow chart of the proposed GA-based optimization model in this study.

TABLE I: THE $3^{\text {RD }}$ ORDER MULTIPLE FEEDBACK LOW PASS FILTER DESIGN’S VARIABLES

\begin{tabular}{|c|c|c|}
\hline Component & Minimum & Maximum \\
\hline R1(Ohm) & $10 \mathrm{~K}$ & $50 \mathrm{~K}$ \\
\hline R2(Ohm) & 800 & $2 \mathrm{~K}$ \\
\hline R3(Ohm) & 500 & 1500 \\
\hline R4(Ohm) & $1 \mathrm{~K}$ & $10 \mathrm{~K}$ \\
\hline R5(Ohm) & 800 & $2 \mathrm{~K}$ \\
\hline R6(Ohm) & $1 \mathrm{~K}$ & $10 \mathrm{~K}$ \\
\hline $\mathrm{C} 1(\mathrm{~F})$ & $5 \mathrm{n}$ & $50 \mathrm{n}$ \\
\hline $\mathrm{C} 2(\mathrm{~F})$ & $100 \mathrm{n}$ & $500 \mathrm{n}$ \\
\hline
\end{tabular}

TABLE II: THE OUTPUT SPECIFICATION FOR $3^{\text {RD }}$ ORDER MULTIPLE FEEDBACK LOW PASS FILTER DESIGN

\begin{tabular}{|c|c|c|c|c|}
\hline Output & Minimum & Maximum & Optimization & Weight \\
\hline Gain $(\mathrm{dB})$ & 8 & 10 & Maximize & 1 \\
\hline $\begin{array}{c}\text { Pass-band } \\
\text { Ripple (dB) }\end{array}$ & 0 & 0.3 & Minimize & 1 \\
\hline $\begin{array}{c}\text { Cuttoff } \\
\text { Frequency } \\
(\mathrm{Hz})\end{array}$ & 1500 & 1600 & Maximize & 10 \\
\hline
\end{tabular}

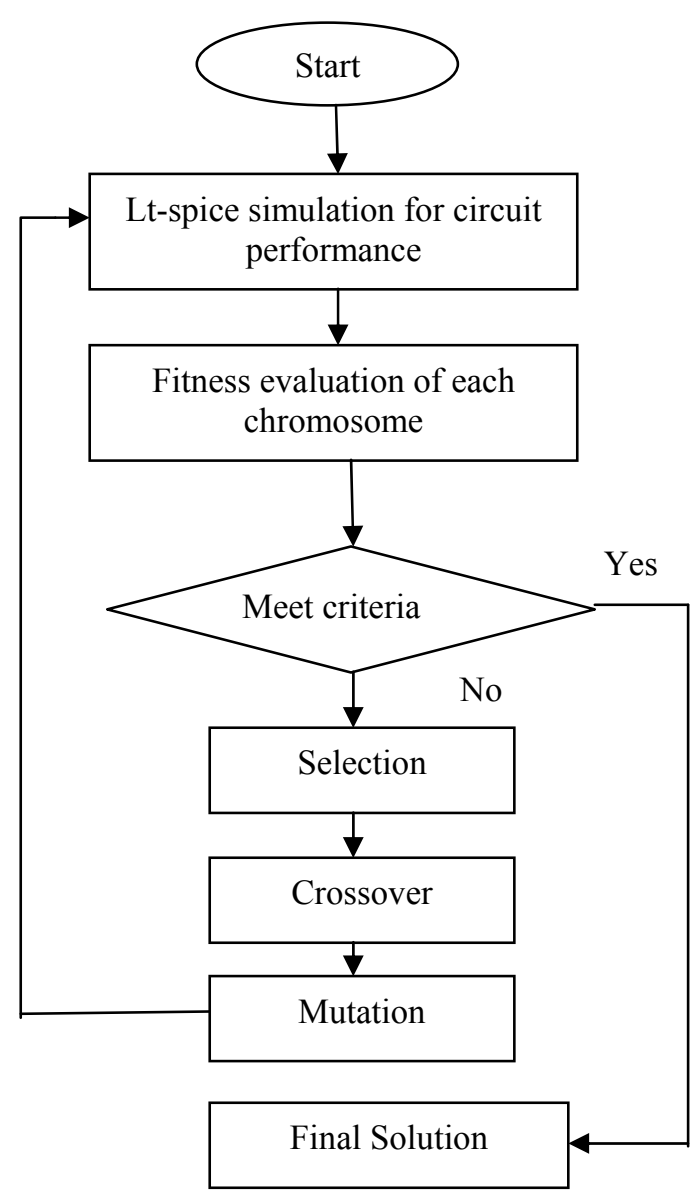

Fig. 2. Flow chart of the basic genetic algorithm.

\section{A. Randomly Generated Chromosomes}

Firstly, a population of 50 chromosomes is randomly generated using Matlab software. Each chromosome comprises of genes that represent the design variables. Then, 
the performance of each chromosome is simulated using LTspiceIV software to obtain the values of output gain, cut-off frequency and ripple. These values are compared to the targeted specification value whereby a fitness evaluation function is developed to measure the fitness of each specification using the normalized weighted sum approach. The optimization for each specification will be considered in the termination criteria. As long as the termination criterion is not met, the chromosomes will be reproduced through the genetic operations of crossover and mutation. The probability for crossover and mutation are based on the fitness obtained whereas the genes to undergo crossover and mutation are randomly selected. The simulation using LTspiceIV is repeated until the termination criterion is achieved. In this study, there are eight design variables (as shown in Table I) to be optimized and three specifications (displayed in Table II) to be achieved.

\section{B. Fitness Evaluation}

After 50 sets of simulation results have been gathered, the chromosome fitness is obtained by executing fitness evaluation with respect to each specification. Equations (1-3) show the fitness assignment function based on each specification where $F_{1}, F_{2}$, and $F_{3}$ refers to the fitness assignment function for gain, pass-band ripple and cutoff frequency respectively. In the evaluation, chromosome is given a penalty value, $\alpha$, when the gain, cutoff frequency and pass-band ripple exceed or less than the specified range. Therefore, if the output specification is not achieved within the specified range the chromosome's fitness will be poor. In order to get the overall fitness for the chromosome, a normalized weight-sum approach is used to sum up all the specification fitness and reduce the possible bias caused by different specification. The overall fitness is given in equation (4) whereby the minimization of total fitness value is targeted. In this case, the lower the fitness value, the better the solution is.

$$
\begin{gathered}
F 1=\left\{\begin{array}{c}
\alpha+(\text { gain }-10), \text { if gain }>10 \\
\alpha+(8-\text { gain }), \text { if gain }<8 \\
10-\text { gain, if } 8 \leq \text { gain } \leq 10
\end{array}\right. \\
F 2=\left\{\begin{array}{c}
\alpha+(\text { ripple }-0.3), \text { if ripple }>0.3 \\
\alpha+(0-\text { ripple }), \text { if ripple }<0 \\
\text { ripple }-0, \text { if } 0 \leq \text { ripple } \leq 0.3
\end{array}\right. \\
F 3=\left\{\begin{array}{c}
\alpha+(\text { cutoff }-1600), \text { if cutoff }>1600 \\
\alpha+(1500-\text { cutoff }), \text { if cutoff }<1500 \\
1600-\text { cutoff, if } 1500 \leq \text { cutoff } \leq 1600
\end{array}\right. \\
F_{\text {overall }}=\sum_{i=1}^{i=3} \frac{F_{i}}{F_{\text {iaverage }}}
\end{gathered}
$$

where;

$$
F_{\text {overall }}=\text { Total fitness of individual; }
$$

$$
\begin{gathered}
F_{i}=\text { Fitness of the } i^{\text {th }} \text { specification; } \\
F_{\text {iaverage }}=\text { Average value of the } i^{\text {th }} \text { specification in the } \\
\text { initial GA generation. }
\end{gathered}
$$

\section{Termination}

The termination criterion happens when the desired output specifications are achieved. Whenever the termination criterion is not fulfilled, the chromosomes will undergo several genetic reproduction operators repeatedly until the final result that fulfills the specifications is obtained [8]. The best chromosome's specification of each GA operation will be compared to the desired specification in Table II in terms of gain, cut-off frequency and pass-band ripple. Chromosomes with smallest fitness evaluation value have higher opportunity to be chosen for genetic crossover and mutation for next iteration. The crossover operator inter-exchanges and combines features between two chromosomes whereas mutation operator will mutate some feature or genes of the selected chromosome with mutation probability. The process of crossover and mutation generate new offspring, producing new candidate solutions for the next generation. With the new offspring, the cycle of LTspiceIV simulation and fitness evaluation are then repeated [8]-[9].

\section{RESUlTS AND DISCUSSION}

The 3rd order multiple feedback low pass filter circuit design is optimized using MATLAB and simulated using LTspiceIV. 50 chromosomes are kept in the population of every generation. In this case study, the genetic reproduction undergoes 10 generation cycles until the results converged to the optimized output specifications that fulfill the design requirements. Table III shows the optimized design variables for the 3 rd order multiple feedback low pass filter

TABLE III: THE OPTIMIZED DESIGN VARIABLES USING
\begin{tabular}{|c|c|}
\hline Design Variables & Value \\
\hline R1 (Ohm) & $20 \mathrm{k}$ \\
\hline R2 (Ohm) & 876 \\
\hline R3 (Ohm) & 735 \\
\hline R4 (Ohm) & $4 \mathrm{k}$ \\
\hline R5 $(\mathrm{Ohm})$ & 1029 \\
\hline R6 (Ohm) & $6 \mathrm{k}$ \\
\hline C1(F) & $6 \mathrm{n}$ \\
\hline C3(F) & $168 \mathrm{n}$ \\
\hline
\end{tabular}

In addition, the best overall fitness obtained in every GA generation is depicted in the Table IV. As observed, the degradation of fitness value from the 1 st to the 6th generations indicates improvement of candidate solutions. The search starts to converge from the $7^{\text {th }}$ generation to the $10^{\text {th }}$ generation.

GA has successfully optimized filter design for gain, cut-off frequency and ripple. Based on the optimized design variables in Table III, Table $\mathrm{V}$ shows the optimized output specifications obtained from LTspiceIV simulation.

By comparing the results to the required specifications in Table II, all output specifications are fulfilled. The gain was maximized to $8.8194 \mathrm{~dB}$ and the pass-band ripple was minimized to $0.087296 \mathrm{~dB}$. As for the cut-off frequency, the 
output obtained is nearly $1550 \mathrm{~Hz}$ which is within the specification range and it is proximately close to the targeted value of $1600 \mathrm{~Hz}$. The overall results satisfied the desired filter performance.

TABLE IV: THE BEST Found OVERALl FitNESS VALUE FOR EACH GA GENERATION

\begin{tabular}{|c|c|}
\hline Number of generation & Fitness value \\
\hline 1st & 22.05159 \\
\hline 2nd & 16.02045 \\
\hline 3rd & 14.07516 \\
\hline 4th & 9.740472 \\
\hline 5th & 9.740472 \\
\hline 6th & 8.898189 \\
\hline 7th & 2.027813 \\
\hline 8th & 2.027813 \\
\hline 9th & 2.027813 \\
\hline 10th & 2.027813 \\
\hline
\end{tabular}

TABLE V: FinAl RESUlts OBTAINED FOR $3^{\text {RD }}$ ORDER MULTIPLE FEEDBACK LOW PASS FILTER

\begin{tabular}{|c|c|}
\hline Output & Target \\
\hline Gain $(\mathrm{dB})$ & 8.81954 \\
\hline Pass-band Ripple $(\mathrm{dB})$ & 0.087296 \\
\hline Cut-off frequency $(\mathrm{Hz})$ & 1549.92 \\
\hline
\end{tabular}

\section{CONCLUSION}

Based on the results, the optimized 3rd order multiple feedback low pass filter managed to meet the specifications required. With the combination of GA and LTspiceIV, the gain is maximized, the ripple is minimized and the cut-off frequency is obtained within the specification range. Despite the capability of GA in optimizing the parameters, there are still rooms for improvement to further increase the gain to 10 $\mathrm{dB}$ and maximize the cut-off frequency to $1600 \mathrm{~Hz}$. Future research on greedy search and GA hybridization can be further explored for better filter design optimization. Besides, additional experiments can be tested on more complicated circuits which involve more constraints and parameters.

\section{REFERENCES}

[1] K. L. Cheng and S. C. Neoh, "Genetic algorithm-based optimization in 4th order sallen key high pass filter," in Proc. 2012 4th Asia Symposium on Quality Electronic Design (ASQED), Penang , 2012, pp. 174-177.

[2] Oxford Dictionaries. [Online]. Available: http://oxforddictionaries.com/definition/optimize

[3] J. H. Holland, "Outline for a logical Theory of adaptive systems," Journal of the ACM (JACM), vol. 9, pp. 297-314, 1962.

[4] Y. Wang, V. Ojleska, Y. Jing, T. K. Gugulovska, and G. M. Dimirovski, "Short term load forecasting: A dynamic neural network based genetic algorithm optimization," in Proc. $14^{\text {th }}$ International Conference on Power Electronics and Motion Control, 2010, pp. 157-161.

[5] T. Back and H. Schwefel, "Evolutionary computation: An overview," in Proc. IEEE International Conference on Evolutionary Computation, 1996, pp. 20-29.

[6] D. Self, Electronic Crossover Design, Focal Press, 2011.

[7] S. C. Neoh, A. Marzuki, N. Morad, C. P. Lim, and Z. A. Aziz, "An interactive genetic algorithm approach to MMIC low noise amplifier design using a layered encoding structure," in Proc. IEEE Congress on Evolutionary Computation, Hong Kong, 2008, pp 1571-1575.

[8] G. liu and J. Chen. "The application of genetic algorithm based on Matlab in function optimization," in Proc. International Conference on Electrical and Control Engineering (ICECE), Yichang, China, 2011, pp. 5034-5037.

[9] T. Kugelstadt, Active Filter Design Techniques, Texas Instrument, 2008.

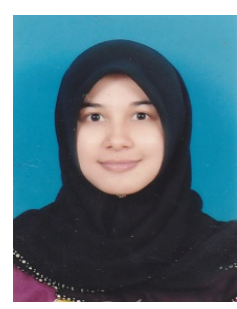

A. A. Wan was born on 22 June 1990 at Nibong Tebal, Pulau Pinang. Currently she is still pursuing her Bachelor Degree in the School of Microelectronic Engineering at University Malaysia Perlis (UniMAP), Malaysia

She was an intern at the IC design Department of Emerald System Sdn. Bhd in 2012. Her research interests include IC Design, Parameter Optimization, and Genetic Algorithm.

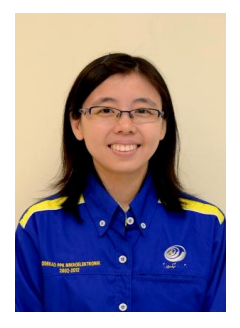

S. C. Neoh received her B. Tech. degree in quality control and instrumentation from the School of Industrial Technology, Universiti Sains Malaysia (USM) in 2003 and Ph.D. degree in the School of Electrical and Electronic Engineering, USM, Nibong Tebal in 2009. Her research interests include evolutionary computing, circuit design optimization, production planning, intelligent manufacturing systems, operations management, and decision support systems. 